\title{
Antenatal manifestations of mitochondrial disorders
}

Mariana Silva Vide Tavares

Serviço de Obstetrícia,

Hospitais da Universidade de Coimbra 


\begin{abstract}
Introduction: Mitochondria are crucial organelles of the cell and its main function is to produce and provide energy for cellular metabolism in most organs and tissues, through a process called oxidative phosphorylation. Mitochondrial respiratory chain diseases are a heterogeneous group of pathologies due to genetic mutations affecting mitochondrial energy production. The estimated incidence is approximately 1:10.000 births. A defect of oxidative phosphorylation can theoretically lead to any symptoms, in any organ or tissue, at any age or even before birth.
\end{abstract}

Objective: To identify the frequency of antenatal manifestations of mitochondrial respiratory chain disorders, characterize the phenotypes and identify possible associations between mitochondrial respiratory chain disorders and a more specific and earlier manifestation or diagnostic disclosure of these diseases.

Methods and subjects: The files of a group of pediatric subjects with mitochondrial respiratory chain disease identified in the first decade of life at the Laboratory of Biochemistry Genetics-Centre for Neuroscience and Cell Biology and Faculty of Medicine, University of Coimbra, in a period of 10 years (2000-2010) were retrospectively reviewed. The results of prenatal and birth history were compared with a control group, which included 2 healthy infants matching patients by the month and year of birth, for each case of a child with mitochondrial disease diagnosis. 
Results [patients (group A)(n=45) versus controls (group B)(n=90)]: Maternal age was (mean \pm standard deviation) $28.4 \pm 6.5$ and $28.3 \pm 4.7$ years, respectively. Gestacional age at delivery was $38.8 \pm 1.7$ and $38.5 \pm 2.6$ weeks. The ratio of children gender (male: female) was 0.73 vs 1 and birth weight at delivery was $2923.2 \pm 554.2$ vs $3246.6 \pm 460.2$ grams $(p=0.001)$. Concerning family history, neurologic diseases were found in 9 vs 5 cases and genetic diseases in 2 vs 0 . Fifteen pregnancies of group A were considered abnormal. The anomalies observed were: intrauterine growth restriction $(n=9)$, oligohydramnios $(n=2)$, preterm delivery $(n=2)$, fetal anemia/anasarca $(n=1)$, maternal infection with varicella $(n=1)$, poor fetal movements $(n=1)$. Neonatal morbidity is significantly higher (5 fold) in group A $(p<0.001)$.

Conclusions: Intrauterine growth restriction was the most frequent antenatal feature observed. Some patients presented isolated antenatal manifestations, such as oligohydramnios and decrease fetal movements. The present work is a relevant contribution, but more studies are needed, in order to study mitochondrial physiology and activity in embryological development for the assessment of the mitochondrial disease development in fetal life. This knowledge will probably contribute to improve our ability to suspect and/or diagnose a mitochondrial respiratory chain disorder from an antenatal manifestation.

\section{Key-words}

Mitochondrial respiratory chain disease; nuclear DNA; mitochondrial DNA; antenatal manifestations; intrauterine growth restriction 


\section{Introduction}

Mitochondria are crucial organelles of the cell and its main function is to produce and provide energy for cellular metabolism in most organs and tissues, through a process called oxidative phosphorylation (OXPHOS). This process occurs in a multienzymatic system located in the mitochondrial inner membrane, - the mitochondrial respiratory chain (MRC), which is encoded by mitochondrial and nuclear DNA (mtDNA and nDNA) and is composed of five polypeptides (I-V) and two intermediate electron carriers - coenzyme Q or ubiquinone and cytochrome C. Apart from complex II, which is exclusively encoded by nDNA, complexes I, III, IV and V are encoded by both nDNA and mtDNA (Van den Heuvel and Smeitink, 2001).

Mitochondrial respiratory chain diseases (MRCD) are a heterogeneous group of pathologies due to genetic mutations affecting mitochondrial energy production. The estimated incidence of these diseases is approximately 1:10.000 births (Van den Heuvel and Smeitink, 2001). A recent epidemiological study has shown that the lowest prevalence at birth is about 1:20.000, indicating MRCD as the most common group of inherited metabolic disease (Andrew et al., 2004; Schaefer et al., 2004).

The Portuguese data from "Centro" region was obtained between 1997-2006 in a population of a tertiary pediatric hospital, revealing an incidence of 1.5:10.000 and a prevalence of 5.4:100.000 in children under 10 years (Diogo et al., 2009).

The MRCD may be associated to any mode of inheritance: sporadic, autosomal recessive, dominant, X-linked or maternal inheritance. Alterations in nDNA follow Mendel's laws and can be grouped into two categories: mutations in nuclear-encoded respiratory chain subunits and mutations affecting assembly and maintenance protein genes. On the other hand, mutations of mtDNA can be classified as: point mutations, deletions/duplications and reductions in the copy 
number (depletion) (Munnich et al., 2001). The peculiarities of the mtDNA, such as maternal inheritance, heteroplasmy, tissue distribution and threshold effect are somewhat responsible for the heterogeneity observed in MRCD (Grazina, 2004).

In theory, a defect of OXPHOS can lead to any symptom, in any organ or tissue, at any age or even before birth (Munnich and Rustin, 2001), due to the bigenomic origin of the MRC components (Van den Heuvel and Smeitink, 2001; Munnich, 2000). Although with possible multiple clinical presentations, MRC disorders should be suspected when there is an unexplained combination of neuromuscular disease and/or neuromuscular symptoms involving different organs and systems with progressive course (Munnich, 2000; Munnich and Rustin, 2001 and Munnich et al., 2001). In the newborn, the most common manifestations are neurological, involving the central nervous system with trunk hypotonia and severe acidotic coma, convulsions, apnea and poor suction; muscular with hypotonia, muscle atrophy and hypotonia, poor head control; heart disease with hypertrophic cardiomyopathy and renal involvement with Fanconi syndrome (Munnich, 2000; Munnich and Rustin, 2001). Some authors consider that around $20 \%$ of the newborns will be small for gestational age, illustrating the importance of this antenatal expression of the disease (Munnich and Rustin, 2001; Munnich et al., 2001).

The aim of the present study is to evaluate the frequency of antenatal manifestations of MRC disorders in a population of children carrying genetic alterations, to characterize the manifestations and identify possible associations between MRC disorders and a more specific and earlier manifestation and/or diagnostic disclosure of these diseases. 


\section{Design and methods}

The files of a group of subjects in pediatric age, with mitochondrial respiratory chain disease identified in the first decade of life at the Laboratory of Biochemistry Genetics - Centre for Neuroscience and Cell Biology and Faculty of Medicine, University of Coimbra, in a period of 10 years (2000-2010) were retrospectively reviewed.

The diagnosis of mitochondrial disease was based on evaluation of enzymatic activity of MRC complexes and genetic analysis with identification of mtDNA mutations (deletions, point mutations, depletion) and nDNA alterations.

The antenatal and neonatal history was obtained from clinical history files of Obstetric Unit of Hospitais da Universidade de Coimbra and Metabolic Diseases Unit, Centre for Child Development "Luís Borges", Pediatric Hospital of Coimbra.

In order to evaluate the significance of the findings, a control group was considered: two healthy infants matching patients by the month and year of birth, from the database of the delivery room of Obstetric Unit of Hospitais da Universidade de Coimbra, for each case of a child with MRCD diagnosis.

The following parameters were evaluated in both patients (group A) and controls (group B): maternal age, maternal and/or family history of pathology (including neuromuscular or cardiac), previous obstetrical history in multiparous women (history of preterm labour and intrauterine growth restriction), complications during actual pregnancy- namely infections (cytomegalovirus, toxoplasmosis, varicella), intrauterine growth restriction (IUGR) - defined as estimated fetal weight below $10^{\text {th }}$ percentile for gestational age (Hadlock), abnormal ultrasound findings, hypertension, preterm delivery (PTD) - defined as delivery before 37 week of gestation, 
fetal death and gestational age (GA) of its occurrence, gestational age at delivery, mode of delivery, complications at delivery, birth weight and Apgar index of the newborn (NB), neonatal morbidity [subdivided in the following categories: respiratory - respiratory distress syndrome (RDS); neurological - hypotonia/poor spontaneous movements; metabolic - hypoglycemia, jaundice, cholestasis and hepatic failure; infectious - sepsis; gastrointestinal - anorexia, vomiting] and neonatal mortality.

Statistical analysis was performed using SPSS 13.0 Statistical Software (SPSS Inc, Chicago, IL) to compare the results between cases and controls. For quantitative variables, the existence of a normal distribution by Kolmogorov-Smirnov Test and homogeneity of variances by Levene's Test was studied. When the results followed a normal distribution and homogeneity of variances was found, a t-Test for comparison of data between the groups was carried out. Whenever the condition of normality underlying quantitative variable under study and/or the condition of homogeneity failed, the nonparametric Wilcoxon-Mann-Whitney Test was used. The qualitative variables were organized in contingency tables and $\chi 2$ or Fisher's exact tests were used to perform association analysis. The strength of the association was evaluated by Pearson's contingency measure. A $p$ value $<0.05$ was considered for defining statistical significance. 


\section{Results}

A total of 45 patients and 90 control subjects were included in this study. Pathogenic mtDNA alterations of the patients included: depletion $(n=7)$, point mutations $(n=4)$, deletions $(n=28)$, deletion and point mutations $(n=1)$. An isolated nDNA variation was present in 1 patient and nDNA alteration plus depletion in 4 patients.

The results obtained from files analysis are described below in a comparative form between the 2 groups created: patients (group A) ( $n=45)$ versus controls (group B) $(n=90)$.

Mean maternal age was $28.4 \pm 6.5$ and $28.3 \pm 4.7$ years, for group A and B, respectively. Gestacional age at delivery was $38.8 \pm 1.7$ and $38.5 \pm 2.6$ weeks, respectively. The ratio of children gender (male:female) was 0.73 vs 1 and birth weight at delivery was $2923.2 \pm 554.2$ vs $3246.6 \pm 460.2$ grams $(p=0.001)$.

One third of mothers vs two thirds were nulliparous. In previous obstetrical history PTD was found in 2 vs 3 cases and IUGR in 1 vs 0.

Concerning family history, neurological diseases were found in 9 vs 5 cases and genetic diseases in 2 vs 0 [One case of Cohen syndrome and other of Ornithine transcarbamylase (OTC) deficiency].

Six children with MRC disorders had consanguineous parents (four in first degree) compared to zero in control group $(p=0.001)$.

Fifteen pregnancies of group A were considered abnormal. The anomalies observed were: IUGR $(n=9)$, oligohydramnios $(n=2)$, PTD $(n=2)$, fetal anemia/anasarca $(n=1)$, maternal infection with varicella $(n=1)$, poor fetal movements $(n=1)$ (Table I). All this anomalies were diagnosed in the third trimester of pregnancy, except the maternal infection by varicella. 
Table I - Gestacional events in MRCD patients and control group.

\begin{tabular}{|c|c|c|c|}
\hline Gestacional events & Patients (n) & Controls (n) & $\begin{array}{l}\text { Statistical } \\
\text { significant }(p)\end{array}$ \\
\hline IUGR & 9 & 3 & 0.003 \\
\hline Oligohydramnios & 2 & 0 & n.s \\
\hline Fetal anemia/anasarca & 1 & 0 & n.s \\
\hline PROM & 1 & 0 & n.s \\
\hline PTD & 2 & 3 & n.s \\
\hline Poor fetal movements & 1 & 0 & n.s \\
\hline Maternal infection with varicella & 1 & 0 & n.s \\
\hline
\end{tabular}

Regarding the type of delivery, 9 vs 16 were born by c-section; 5 vs 8 of those were performed due to fetal distress.

Low Apgar score index, considered as less than 3 at the first minute and less than 7 at 5 minutes of life were found in 1 and 2 newborns with MRC disorders, respectively.

Neonatal morbidity (Table II) is significantly higher ( 5 fold) in group A $(p<0.001)$. 
Table II- Neonatal morbidity in MRCD patients and control group.

\begin{tabular}{|c|c|c|c|}
\hline Neonatal morbidity & Patients $(n)$ & Controls $(n)$ & $\begin{array}{l}\text { Statistical } \\
\text { significant }(p)\end{array}$ \\
\hline Total & 15 & 3 & $<0.001$ \\
\hline \multicolumn{4}{|l|}{ Respiratory } \\
\hline$-\quad$ RDS & 3 & 2 & n.s \\
\hline \multicolumn{4}{|l|}{ Neurological } \\
\hline - Hypotonia/poor spontaneous movements & 5 & 2 & 0.001 \\
\hline - $\quad$ Seizures & 1 & 0 & n.s \\
\hline \multicolumn{4}{|l|}{ Metabolic } \\
\hline - Hypoglycemia & 3 & 0 & n.s \\
\hline - Jaundice & 2 & 0 & n.s \\
\hline - Cholestasis & 2 & 0 & n.s \\
\hline - Hepatic failure & 2 & 0 & n.s \\
\hline \multicolumn{4}{|l|}{ Gastrointestinal } \\
\hline - Anorexia & 2 & 0 & n.s \\
\hline - Vomiting & 2 & 0 & n.s \\
\hline \multicolumn{4}{|l|}{ Infectious } \\
\hline - Sepsis & 2 & 0 & n.s \\
\hline
\end{tabular}

Neonatal mortality was observed in one patient with MRC disorder in the first day of life. The cause of death was respiratory insufficiency by pulmonary hypertension. 


\section{Discussion}

There is limited information on the antenatal manifestations of MRC deficiencies. Yanicostas et al. (2011) and DiMauro and Garone (2011) reflected about development aspects of MRC from fetus to infancy and conclude that little is known about mitochondrial function during fetal life in humans. These authors conclude that intuitively, because of fetal development and its high energy demanding process mitochondria will be in full action, as the most active machinery in delivering cellular consumable energy. However, some experiments with mammals, especially in mouse, have shown that mitochondrial activity varies during fetal life from few and short activity at embryonic mitochondria to active metabolic activity in differentiated cells. In the first trimester of pregnancy, the embryo develops in a low oxygen environment with low levels of biosynthesis, whereas in the third trimester, high oxidative metabolism takes place through the mitochondria to sustain the rapid fetal growth. In conclusion, the mentioned authors' defend that MRC anomalies will only affect fetal life when it relies on newly synthesized mitochondria.

Deletions were the most frequent mtDNA alteration identified in our sample, but other pathogenic mtDNA alterations were found as well as nDNA alterations.

Deletions and duplications are part of the second class of mtDNA alterations - the rearrangements. They are usually sporadic, heteroplasmic and unique, and frequently are caused by de novo rearrangements that arose during oogenesis or early development. No correlation was found between clinical presentation and the nature or extent of rearrangements. Other important characteristic is that deletions increase in proportion over the course of the disease, suggesting that they have a replicative advantage over normal molecules (Munnich and Rustin, 2001; Grazina, 2004). In our sample, there was no evidence of MRC family history but we found a 
higher incidence of neurologic diseases in family relatives and there is higher prevalence (with statistical significance) of first degree consanguineous parents in MRCD patients.

Additionally, mtDNA deletions could be present in close maternal relatives indicating that maternal inheritance may occur. Rare cases were associated with autosomal dominant and recessive multiple DNA deletions (Munnich and Rustin, 2001; Grazina, 2004).

The male/female ratio of 0.73 in group $\mathrm{A}$ is different from those found in literature. Scaglia et al. (2004) and Skadal et al. (2003) describe a male/female ratio of 1.5:1 in groups of 113 and 75 pediatric patients, respectively.

We found that 15 patients had unspecific antenatal manifestations. Intrauterine growth restriction was the most frequent antenatal feature observed. Other isolated antenatal manifestations, such as oligohydramnios and decrease fetal movements were observed, in agreement with findings by Gibson et al. (2008) and Von Kleist-Retzow et al. (2003). However, in our sample, the type and frequency of anomalies were not similar to what is described by those authors, especially when concerning antenatal malformations and multiple organ involvement. Munich et al. (2001) consider that mitochondrial diseases are not commonly regarded as causes of fetal malformation.

As described earlier, Yanicostas et al. (2011) and DiMauro and Garone (2011) affirmed that in the third trimester, high oxidative metabolism takes place through the mitochondria to sustain the rapid fetal growth. This suggests that in a presence of a MRCD, fetal growth can be compromised in the last trimester. In our results, the prevalence of IUGR in group A is relevant and all cases were diagnosed in the last trimester of pregnancy in agreement with the mentioned authors'. 
In our analyses, low birth weight was more frequent in children with MRCD when compared with control group. These results are similar to those described by Von Kleist-Retzow et al. (2003).

Gibson et al. (2008) suggest that the high energy demands of labor, delivery and the early neonatal period increases the vulnerability of children with MRC disorders, because they have an impaired energy production. However, in our sample, the majority of MRCD patients were born by vaginal delivery with satisfactory Apgar score index, not significantly different from controls. Nevertheless, the $9 \mathrm{C}$-sections performed in patients were by fetal distress although there is an apparent lack of association between MRC disorders and the type of delivery.

A higher number of neonates with MRC disorders had significantly more neonatal manifestations when compared to controls $(p<0.001)$. Data in table II shows that the most frequent manifestations were hypotonia/poor spontaneous movements $(p=0.001)$ followed by recurrent hypoglycemia, cholestasis, hepatic failure and sepsis. These results are coincident with those described in the literature (Munnich, 2000; Munnich and Rustin, 2001; Munnich et al., 2001). We have not identified any case of cardiac presentation, frequently described as the main cause of cardiomyopathy in children with MRC disorders.

Maternal age, pregnancy complications as hypertension or fetal deaths, GA at delivery and neonatal mortality were not found to be significantly different between the two groups. These results are similar to those described by Von Kleist-Retzow et al. (2003).

The main limitation of this study is the small number of cases. However, our results are coincident with the limited literature available. Another limitation is the fact that data was retrospectively reviewed from patient's files could lead to the lost of some antenatal information. 
The children observed were in the first decade of life and so the mutations found were probably more frequent with earlier manifestations. Von Kleist-Retzow et al. (2003) in his study conclude that the time course and/or tissue specificity of disease expression rather than the or severity of the enzyme deficiency should predict the antenatal expression pattern of the disease.

Our purpose to identify a possible association between MRC disorders and a more specific and earlier manifestation and/or diagnostic clinical sign was not fully accomplished. Intrauterine growth restriction is the main antenatal manifestation in MRC disorders. However, the possible causes of IUGR are innumerous. Its principal cause is related with placental insufficiency, but it may also be due to a large number of other conditions, such as congenital anomalies, infections and multifactorial anomalies (Graça, 2010). The incidence of MRC disorders in children with IUGR as not been described yet but we suppose that it will be residual. For the reasons exposed, we think that a future multicentric study to assess the real importance of MRC disorders in IUGR is of great importance.

None of the antenatal manifestations found in the present study were typical and/or can be found in all cases of MRC disorders in order to increase clinical suspicious of MRC disorders when found in fetal life. This fact limits our capacity to identify, diagnose and act in antenatal period.

On the other hand, genetic counseling requires exact knowledge of the mode of inheritance. Most disorders occur sporadically, although some are inherited in an autosomal recessive or dominant and maternal inheritance (Munich and Rustin, 2001). Beside the incomplete knowledge of the tissue distribution of MRC disorders, its change during 
development and its quantitative relationship to disease severity, compromises possibility of prenatal diagnosis.

In conclusion, although a great number of MRC diseases do not have antenatal expression, some cases with early neonatal/infant expression can present some manifestations in fetal life. The presence of IUGR and other isolated anomalies should alert clinicians to the possibility of MRCD.

The present work is a relevant contribution, but more studies are needed, in order to study mitochondrial physiology and activity in embryological development for the assessment of the mitochondrial disease development in fetal life. This knowledge will probably contribute to improve our ability to suspect and/or diagnose a mitochondrial respiratory chain disorder from an antenatal manifestation. 


\section{Acknowledgments}

Special thanks to Manuela Grazina PhD, Paulo Moura MD, PhD and Ana Patrícia Domingues MD, MSc for the guidance provided in the elaboration and critical review of this article.

To those responsible by the Laboratory of Biochemistry Genetics - Centre for Neuroscience and Cell Biology and Faculty of Medicine, University of Coimbra, Manuela Grazina PhD; Obstetric Unit of Hospitais da Universidade de Coimbra, Paulo Moura MD, PhD and Metabolic Diseases Unit, Centre for Child Development "Luís Borges" from Paediatric Hospital of Coimbra, Luísa Diogo MD, PhD for the disposal of clinical history files. 


\section{References}

DiMauro S., Garone C. (2011). Metabolic disorders of fetal life: Glycogenesis and mitochondrial defects of the mitochondrial respiratory chain. Seminars in Fetal \& Neonatal Medicine $16: 181-189$

Diogo et al. (2009). Pediatric Mitochondrial Respiratory Chain Disorders in Centro Region of Portugal. Pediatr Neurol 40:315-356

Gibson et al. (2008). Mitochondrial Oxidative Phosphorylation Disorders Presenting in Neonates: Clinical Manifestations and Enzymatic and Molecular Diagnoses. Pediatrics 122:10031006

Graça LM (2010). Restrição de crescimento intra-uterino. In: Medicina Materno-Fetal (4ªedição). Lidel Edições; 40:456-464

Grazina MM (2004). Genoma Mitocondrial e Défice Energético no Diagnóstico das Doenças da Cadeia Respiratória Mitocondrial. Dissertação de Doutoramento.

Munnich A (2000). Defects of the Respiratory Chain. In: Inborn Metabolic Diseases - Diagnosis and Treatment ( $3^{\text {rd }}$ edition). Fernandes J, Saudubray J-M, Van den Berghe (Eds) Springer; 13:159-168.

Munnich A, Rotig A, Cronier-Daire V, Rustin P (2001). Clinical presentation of Respiratory Chain Deficiency. In: Inborn Metabolic Disease - Diagnosis and Treatment (3rd edition). Fernandes J, Saudubray JM, Van den Berghe G (Eds.) Springer 99:2261-2274 
Munnich A, Rustin P (2001). Clinical spectrum and diagnosis of mitochondrial disorders. Am J Med Genetics (Semin. Med. Genet.) 106:4-17

Scaglia et al. (2004). Clinical spectrum, morbidity and mortality of 113 pediatric patients with mitochondrial disease. Pediatrics 114:925-931

Schaefer et al. (2004). The epidemiology of mitochondrial disorders - past, present and future. Biochimica et Biophysica Acta 1659:115-120

Skladal et al. (2003). The clinical spectrum of mitochondrial disease in 75 pediatric patients. Clin Pediatr 42:703-710

Trijbels JMF et al. (1988). Disorders of the mitochondrial respiratory chain: clinical manifestation and diagnostic approach. Pediatrics 148(2): 94-97

Van den Heuvel L, Smeitink J. (2001). The Oxidative phosphorylation (OXPHOS) system: nuclear genes and human genetics diseases. BioEssays 23:518-525

Von Kleist-Retzow et al. (2003). Antenatal manifestations of mitochondrial respiratory chain deficiency. J Pediatr 143: 208-212

Yanicostas et al. (2011). Development aspects of respiratory chain form fetus to infant. Seminars in Fetal \& Neonatal Medicine 16:175-180 